\title{
BMJ Open Post-traumatic stress disorder in UK civilians with traumatic brain injury: an observational study of TBI clinic attendees to estimate PTSD prevalence and its relationship with radiological markers of brain injury severity
}

\author{
Kasim L Qureshi, ${ }^{1}$ Rachel Upthegrove, ${ }^{1}$ Emma Toman, ${ }^{2}$ Vijay Sawlani, ${ }^{3}$ \\ David James Davies, ${ }^{2}$ Antonio Belli ${ }^{4}$
}

To cite: Qureshi KL,

Upthegrove R, Toman E, et al. Post-traumatic stress disorder in UK civilians with traumatic brain injury: an observational study of TBI clinic attendees to estimate PTSD prevalence and its relationship with radiological markers of brain injury severity. BMJ Open 2019:9:e021675. doi:10.1136/ bmjopen-2018-021675

- Prepublication history for this paper is available online. To view these files, please visit the journal online (http://dx.doi org/10.1136/bmjopen-2018021675).

Received 12 January 2018 Revised 22 May 2018 Accepted 23 October 2018

D Check for updates

(c) Author(s) (or their employer(s)) 2019. Re-use permitted under CC BY-NC. No commercial re-use. See rights and permissions. Published by BMJ.

For numbered affiliations see end of article.

Correspondence to Dr Kasim L Qureshi; klq552@alumni.bham.ac.uk, kasimluis.qureshi@nhs.net

\section{ABSTRACT}

Objectives To estimate the prevalence of post-traumatic stress disorder (PTSD) in a large civilian population with traumatic brain injury (TBI), and to assess whether brain injury severity is correlated with PTSD symptoms.

Design Observational, cross-sectional study.

Setting and participants Outpatient clinic in a major UK trauma centre and secondary care hospital. Estimates of PTSD prevalence are based on 171 sampled individuals attending TBI clinic within an 18-month period. Analysis of the relationship between TBI severity and PTSD was performed on the subset of 127 patients for whom injury severity data were also available.

Methods Civilian TBI clinic attendees completed validated self-report questionnaires assessing PTSD (PTSD Checklist Civilian Version (PCL-C)) and other psychiatric symptoms. From this, the prevalence of PTSD was estimated in our cohort. Postresuscitation Glasgow Coma Score and Marshall grade on CT brain scan were recorded as indicators of brain injury severity. A hierarchical regression explored whether TBI severity may predict PTSD scores.

Results A high prevalence of PTSD was estimated $(21 \%$ with PCL-C score >50). Higher Marshall grading displayed a slight negative correlation with PTSD symptoms.

This statistically significant relationship persisted after confounding factors such as depression and postconcussion symptoms were controlled for. Conclusions PTSD and TBI frequently coexist, share antecedents and overlap in their resultant symptoms. This complexity has given rise to conflicting hypotheses about relationships between the two. This research reveals that PTSD is common in civilians with TBI (adding to evidence drawn from military populations). The analysis indicated that more severe brain injury may exert a slight protective influence against the development of PTSD-potentially by disrupting implicit access to traumatic memories, or via overlapping neuropsychiatric symptoms that impede diagnosis. The association suggests that further research is warranted to explore the reuse of routine clinical and neuroimaging data-investigating its potential to predict risk of psychiatric morbidity.
Strengths and limitations of this study

- This study estimates the prevalence of post-traumatic stress disorder (PTSD) using a large and diverse sample of civilians with traumatic brain injury which is representative of the UK clinical population.

- While analysing the link between brain injury and PTSD severity, this study controls for the potentially confounding effect of symptoms due to depression and concussion.

- There is a strong emphasis on using data which can be readily collected in clinical practice (routinely performed CT scans and standardised questionnaires).

- The sampled clinic attendees self-report symptoms at various times after injury. This prevents conclusions being drawn about the timing at which psychiatric symptoms develop, and those who do not access care may be under-represented.

- The study is observational in nature rather than experimental. Conclusions can be drawn regarding brain injury predicting PTSD severity, but this does not necessarily imply a causal link.

\section{INTRODUCTION}

The complex relationship between post-traumatic stress disorder (PTSD) and traumatic brain injury (TBI) presents opportunities to further the understanding of both conditions individually as well as their interplay. PTSD is a common mental health condition with an estimated lifetime prevalence of $7.8 \%{ }^{1}$ Risk is increased by severely distressing experiences such as sexual assault, life-threatening injury or emotional trauma during military service. ${ }^{1}$ Its psychosocial impact is significant, with a high risk of suicidal behaviour in patients with PTSD, ${ }^{2}$ impairments in social and occupational functioning, as well as increased 
utilisation of health services. ${ }^{3}$ The purported aetiology of PTSD involves an antecedent psychologically traumatic event which is deemed severely threatening. The presence of such a stressor is common to diagnostic criteria in the Diagnostic and Statistical Manual 5 (DSM 5$)^{4}$ and the International Classification of Diseases 10 (ICD-10). ${ }^{5}$

A psychologically traumatic event involving physical brain injury can potentially complicate the development of PTSD. Pre-event and postevent amnesia is often a feature of brain injury and concussion and yet the integrity of traumatic memories may also play an important role in the development of the disease. Where memory of an antecedent event is impaired due to traumatic amnesia, it has been proposed that this memory loss may have a potentially protective ${ }^{6}$ or even preventative role $^{7}$ in PTSD development. The lack of intact recollection of a traumatic event may be associated with a failure to develop intrusive, distressing memories which are a hallmark of PTSD.

A varied range of cognitive deficits can result from TBI. This may in fact render patients more vulnerable to the development of PTSD as better premorbid function, with increased cognitive reserve, has been found to be a protective factor. ${ }^{8}$ Further research suggested that both mild TBI (mTBI) and severe TBI may predispose to PTSD even in the presence of amnesia and other cognitive abnormalities. ${ }^{910}$ There are diverse mechanisms by which brain injury may produce cognitive deficits, for example, diffuse axonal fragmentation can disrupt connections between key networks of cortical grey matter. ${ }^{11}$ However, the extent to which neuroimaging and gross structural changes can be linked to the development of PTSD in this patient group is poorly understood. ${ }^{12}$ As a result, uncertainties remain about the neuropathological mechanisms by which TBI and PTSD may be linked, particularly outside of the military/blast injury context.

Large studies exploring the relationships between TBI and PTSD often involve military populations, typically those involved in wars in Afghanistan and Iraq. ${ }^{13}$ Though pragmatic, an approach based on military cohorts is complicated by the potential exposure to multiple psychological stressors aside from the event responsible for TBI. Furthermore research in this population is largely focused on damage attributable to blast injuries or

Table 1 Demographic characteristics and post-traumatic stress disorder (PTSD) Checklist Civilian Version (PCL-C) scores of overall clinic sample $n=171$

\begin{tabular}{|c|c|}
\hline Variable & \\
\hline Age & Range 16-82 years; median 38; IQR 32 \\
\hline Sex & F: 37 (22\%), M: 134 (78\%) \\
\hline Ethnicity & $\begin{array}{l}\text { White } 131(77 \%) \text {, African Caribbean } 6 \\
(4 \%) \text {, Asian } 18(11 \%) \text {, Mixed } 8(5 \%) \text {, } \\
\text { other } 8(5 \%)\end{array}$ \\
\hline $\begin{array}{l}\text { PTSD } \\
\text { (PCL-C score/84) }\end{array}$ & Range 5-84; mean 34.46; SD 18.12 \\
\hline
\end{tabular}

other mechanisms quite specific to military combat. ${ }^{13} 14$ In contrast, civilian TBI is most commonly due to falls, vehicle crashes and assaults (as well as a varied range of other mechanisms). ${ }^{15}$ These mechanisms may result in qualitatively differing patterns of brain damage and psychological trauma, limiting the extent to which findings from specific military studies can be generalised to the civilian populace.

The relationship between the severity of brain injury and the development of PTSD remains controversial, with mixed findings in patients with mild versus severe injury. ${ }^{7-10}$ As a result, some studies have focused on mTBI in order to explore the effect on PTSD. A systematic review of such studies ${ }^{16}$ has highlighted marked heterogeneity of study design which obscures the relationship between the conditions. Drawing a distinction between mTBI and more severe injury may introduce an artificial dichotomy onto the spectrum of brain injury, potentially limiting our understanding of the relationship with PTSD.

This current study aimed to explore the relationship between brain injury factors and PTSD symptoms in a large civilian, outpatient population-while controlling for confounding variables. This has been conducted with the objectives of estimating the prevalence of PTSD, and assessing whether indicators of TBI severity predict PTSD symptom levels.

\section{METHODS}

Data were collected prospectively between December 2013 and June 2015 from patients attending an outpatient TBI clinic at the Queen Elizabeth Hospital in Birmingham-a major trauma centre in the UK. This included processes to ensure participants provided informed consent for their clinical data to be stored in database form, and for anonymised information to be used for the purposes of research.

Admission records were interrogated to record demographic details and best postresuscitative Glasgow Coma Score. ${ }^{17}$ Patients completed a battery of self-report questionnaires, including: health-related quality of life (Quality of Life after Brain Injury (QOLIBRI) ${ }^{18}$ ), postconcussion symptoms (PCS) (Rivermead PCS Questionnaire ${ }^{19}$ ), depression (Patient Health Questionnaire $(\text { PHQ9 })^{20}$ ) and PTSD severity (PTSD Checklist Civilian Version $\left.(\text { PCL-C })^{21}\right)$. Exclusion criteria were: attendance due to non-traumatic pathology, chronic subdural haematoma or declining to provide informed consent. Additionally, participants could not be included if required data for the analysis were not available (as reported in the Results section).

PCL-C is a measure of PTSD symptoms adapted from the military questionnaire use in a civilian population ${ }^{2122}$ and scores can range from 17 (minimal symptoms) to 85 . Two cut-off levels are established to estimate PTSD prevalence using PCL-C scores: scores $<50$ have been regarded as a suitable diagnostic estimate in the mTBI population, and scores $<44$ have been validated based on studies in 
Table 2 Proportion of patients with traumatic brain injury of differing severities based on the best postresuscitation Glasgow Coma Scale (GCS) and Marshall grade $\mathrm{n}=127$

\begin{tabular}{llllll}
\hline GCS & $\begin{array}{l}61 \% \\
\text { (mild: 13-15) }\end{array}$ & & $\begin{array}{l}13 \% \\
\text { (moderate: 9-12) }\end{array}$ & $\begin{array}{l}26 \% \\
\text { (severe: 3-8) }\end{array}$ \\
Marshall grade & $\begin{array}{l}\text { 16\% } \\
\text { (grade 1) }\end{array}$ & $\begin{array}{l}56 \% \\
\text { (grade 2) }\end{array}$ & $\begin{array}{l}3 \% \\
\text { (grade 3) }\end{array}$ & $\begin{array}{l}1 \% \\
\text { (grade 4) }\end{array}$ & $\begin{array}{l}24 \% \\
\text { (grade 5-6) }\end{array}$ \\
\hline
\end{tabular}

populations in which PTSD symptoms are anticipated to be high. ${ }^{23}$ Prevalence estimates were recorded at both thresholds in this study, as use of either can be justified based on the limited prior research in civilian populations. ${ }^{24}$ While estimating prevalence necessitates use of dichotomous cut-offs, the possible associations between PTSD symptom severity and TBI severity may occur below these thresholds. As such, PCL-C scores were treated as a continuous variable in the regression analysis described below. This also reduces the need for multiple comparisons at different cut-off thresholds.

Two relatively objective indicators brain injury severity were also recorded: best Glasgow Coma Scale (GCS) following initial resuscitation, and classification of admission CT brain scan using the Marshal injury burden stratification score. ${ }^{17}$ Rather than basing the analysis on immediate GCS on admission (which can also indicate TBI severity), using the best GCS rating has been found to be a better predictor of long-term functional and cognitive outcomes ${ }^{25} 26$ and this is likely to be relevant to long-term mental health. Marshall grades are defined as: 1-no visible pathology; 2-cisterns present with midline shift $<5 \mathrm{~mm}$ and/or lesion densities present; $3-$ cisterns compressed/absent with midline shift 0-5 mm; 4-diffuse injury with midline shift $>5 \mathrm{~mm}$; 5 -any lesion evacuated surgically; and 6-high or mixed-density lesions $>25 \mathrm{~cm}^{3}$ not surgically evacuated. Grades 5 and 6 were grouped together for the purposes of this analysis, as the progression from one to the other does not necessarily represent an increase in severity. GCS was classified into three severity levels: mild (13-15), moderate (9-12) and severe (3-8). Marshall grade and GCS were assessed

Table 3 Descriptive statistics of the group included in hierarchical regression $n=127$

\begin{tabular}{lcll}
\hline Variable & Range & Median & IQR \\
\hline $\begin{array}{l}\text { Quality of life } \\
\text { (QOLIBRI-\%) }\end{array}$ & $33-100$ & 59 & 29 \\
$\begin{array}{l}\text { Concussion symptoms } \\
\text { (Rivermead PCS) }\end{array}$ & $0-60$ & 24 & 26.25 \\
$\begin{array}{l}\text { Depression symptoms } \\
\text { (PHQ9) }\end{array}$ & $0-26$ & 7 & 15.5 \\
$\begin{array}{l}\text { PTSD symptoms } \\
\text { (PCL-C) }\end{array}$ & $17-85$ & 25 & 28 \\
\hline
\end{tabular}

PCL-C, PTSD Checklist Civilian Version; PCS, postconcussion symptom; PHQ9, Patient Health Questionnaire; PTSD, posttraumatic stress disorder; QOLIBRI, Quality of Life after Brain Injury. by the neurosurgery team involved in the participants' care. As GCS was recorded as part of routine practice by the attending team, it was not within the scope of this research to formally audit this or to assess inter-rater reliability.

A hierarchical multiple regression analysis was performed using Statistical Package for the Social Sciences, V.11 for Windows (SPSS) to determine whether brain injury features (GCS and Marshall grade) were statistically significant predictors of PTSD scores (dependent variable), even when controlling for age, sex, quality of life, concussion symptoms and depression as potential confounding factors. This type of analysis was used due to the a priori hypothesis that brain injury severity may predict some risk of psychiatric morbidity. This thereby justified a qualitative distinction to be drawn between the confounding variables (first stage of the regression analysis) and the potential predictors (second stage).

\section{Patient and public involvement}

Patients and public were not directly involved in the development of this study.

\section{RESULTS}

To produce estimates of PTSD prevalence in this cohort, 171 participants were included (as their full PCL-C scores were available), $79 \%$ were men and the median age was 38. See table 1 for sample description.

Using PCL-C cut-off score $>50$, the prevalence of PTSD was $20.6 \%$; using the lower threshold (score $>44$ ), prevalence was $31.6 \%$.

Brain injury severity data were not available for all of the participants described above, due to incomplete records. Hierarchical multiple regression was performed based on those 127 participants who completed questionnaires, had CT head scan results available and their admission GCS recorded (see table 2). The 44 participants excluded (due to missing data) did not differ significantly in demographic or injury characteristics, nor in PTSD, depression or PCS scores.

Uncorrected exploratory correlations conducted within the regression group $(\mathrm{n}=127)$ suggested that PCSs $(\mathrm{r}=0.70)$ and depression $(\mathrm{r}=0.76)$ were moderately positively correlated with PTSD severity $(\mathrm{p}<0.01)$.

A two-level hierarchical regression analysis was performed (see table 3), with PTSD severity (PCL-C score) as the dependent variable. Model assumptions were tested and met. The first level of the regression consisted of potential predictors of PTSD score which 


\begin{tabular}{llll} 
Table 4 Hierarchical regression models & & \\
\hline Potential predictor & Coefficient beta & B $\mathbf{( 9 5 \%} \mathbf{C l})$ & B SE \\
\hline Model 1 & & & \\
Sex & -0.10 & $-0.47(-6.22$ to 5.28$)$ & 2.90 \\
Age & -0.03 & $-0.03(-0.16$ to 0.10$)$ & 0.06 \\
QoL & 0.13 & $0.15(-0.07$ to 0.36$)$ & 0.11 \\
Concussion symptoms & 0.23 & $0.28(-0.06$ to 0.62$)$ & 0.17 \\
Depression symptoms & 0.65 & $1.57(0.97 \text { to } 2.17)^{\star}$ & 0.30 \\
Model 2 & & & \\
Sex & -0.01 & $-0.51(-6.13$ to 5.11$)$ & 2.84 \\
Age & -0.03 & $-0.03(-0.16$ to 0.09$)$ & 0.06 \\
QoL & 0.13 & $-0.15(-0.07$ to 0.36$)$ & 0.11 \\
Concussion symptoms & 0.18 & $0.22(-0.12$ to 0.56$)$ & 0.17 \\
Depression symptoms & 0.69 & $1.67(1.08 \text { to } 2.26)^{\star}$ & 0.30 \\
GCS† (mild/moderate/severe) & -0.08 & $-1.86(-4.67$ to 0.96$)$ & 1.42 \\
Marshall grade† & -0.12 & $-1.73(-3.45 \text { to } 0.01)^{\star}$ & 0.87 \\
\hline
\end{tabular}

*Statistically significant $p<0.05$.

†Second level of hierarchical regression (routinely recorded brain injury factors).

GCS, Glasgow Coma Scale; QoL, quality of life.

may be confounding factors, specifically age, sex, depression scores (PHQ9), PCSs (Rivermead) and quality of life (QOLIBRI). The second level contained GCS and Marshall grade (see table 4).

At the first level, depression and other potential confounders contribute significantly to the model, $F$ $(5,121)=35.59, \mathrm{p}<0.01$, accounting for $57.9 \%$ of the variance in PTSD severity, with depression the only individual significant factor. The second level including Marshall grade added a modest but statistically significant contribution to PTSD severity $-F(7,119)=28.06, \mathrm{p}<0.05$. In contrast, GCS was not a statistically significant predictor of PTSD severity when other potential confounders were controlled for.

\section{DISCUSSION}

These findings reveal a high level of PTSD symptoms in the civilian TBI clinic population. Dependant on diagnostic threshold used, estimated prevalence of PTSD is between $20.6 \%$ and $31.6 \%$, which is in keeping with previous studies of smaller cohorts. ${ }^{9}$ Furthermore, this is in keeping with findings from military populations showing associations between even mTBI and PTSD. ${ }^{16} 23$ Depression is significantly correlated with PTSD severity. This is plausible given their symptomatic overlap, the tendency of stress to trigger both depressive episodes and PTSD, and it is in keeping with the high level of comorbidity between the two conditions. ${ }^{27}$ Nonetheless, even when depression and other factors are controlled for, Marshall grade is a statistically significant predictor of the variance in PTSD scores. More severe radiological injury burden (based on higher Marshall grade) is associated with less severe PTSD scores. GCS was not a significant correlate of PTSD severity. This reflects the possibility that a conventional distinction between mild, moderate and severe brain injury based purely on postresuscitative GCS, may not reflect the particular factors that predispose toward psychiatric morbidity. Further study is required to explore whether there is a relationship between altered GCS and PTSD in other settings.

The current results suggest that PTSD is common in this large cohort and that routinely collected radiological data may be of use in identifying those at greatest risk of severe PTSD symptoms. The strengths of this study include a pragmatic emphasis on tools which can be employed in routine clinical practice-review of CT scans, GCS levels and self-report questionnaires. Different criteria for PTSD have been used in previous research (using the ICD-10, DSM 4 and DSM 5) each with subtly different emphases. The PCL-C is based on established diagnostic features and can be reliably administered in the clinic setting, thereby enabling comparisons in the wider literature. $^{28}$

This study has attempted to isolate the relationship between PTSD and TBI from confounding factors. Diagnostic confusion can arise when the damage associated with brain injury results in a neuropsychiatric syndrome which overlaps with PTSD even if the initial injury has not featured severe psychological trauma. ${ }^{8}$ This PCS and PTSD potentially both include features such as irritability, and both conditions can be associated depressed mood. ${ }^{10}$ Such potential for overlap at the symptom level introduces the possibility that the co-occurrence of PTSD, depression and PCS may be overestimated. Furthermore, 
some mild cognitive deficits are associated with PCS which may increase vulnerability to PTSD as previously discussed. In this study, controlling for PCSs within the regression analysis served to partially mitigate against this potential source of confusion.

Inevitably, certain limitations apply to the approach presented. A brief survey inevitably produces less precise estimates of prevalence of PTSD than a full psychiatric assessment. However, prior research suggests a high rate of psychiatric symptoms and that PTSD may be underdiagnosed in this group, ${ }^{16}$ so measuring symptom severity may highlight those for whom psychiatric review would be beneficial and could lead to diagnosis. Broader concerns about self-report measures may apply-whether this manifests as patients denying the severity of their symptoms, or overstating them in the hope of receiving more support. In spite of this, the PCL-C has been found to be reliable across comparable populations. ${ }^{28}$

The outpatient sample taking part in this study represents a large and well-categorised civilian group in a real-world hospital setting; however, some factors may limit its generalisability to the wider TBI population. There is potential for selection bias in favour of patients with more persistent symptoms, as those attending the clinic are more likely to have enduring neuropsychiatric symptoms which justify their attendance. Conversely, patients with more severe injuries may have cognitive deficits that render them unable to complete the necessary questionnaires for inclusion, or they may be in inpatient settings that make clinic attendance less likely. The potential also exists for the severity of TBI to be underestimated through the use of best GCS score after resuscitation, rather than the use of initial GCS on admission. However, this compromise improves the ability of GCS to predict long-term outcomes. ${ }^{25} 29$ In spite of the majority of the cohort consisting of mTBI, the sample contains a wide range of injury severity levels, which partially serves to mitigate against a systematic bias of this type. The use of routinely collected clinical brain injury data (GCS, CT scan findings) is advantageous in that it is readily available and quite objective in nature, but the fact that such data are/may be recorded by different clinical teams (without specific training for the purpose of this study) has the potential to reduce inter-rater reliability.

This study sought to characterise this particular civilian TBI cohort, as data from similar large populations are relatively limited. However, the absence of a control group does limit the extent to which one can meaningfully speculate about the neural mechanisms by which TBI and PTSD may be linked. To elucidate this in future, studies including a control group of participants with extracranial trauma may be valuable to isolate the effect of brain damage from other aspects of psychological trauma associated with injury and hospitalisation. Finally, the majority male sample may be typical of TBI sufferers; however, this may be less representative of the wider civilian PTSD cohort. This study included TBI both with and without structural changes identified on CT. A full understanding of the links between acquired brain injury and psychiatric symptoms will require the location of any overt injury to be taken into account, although this was beyond the scope of the analysis presented.

The high prevalence of PTSD found in this study provides an important epidemiological estimate within the UK civilian population. These prevalence findings are in accordance with research in populations who have suffered general trauma (including extracranial injury) such as those involved in motor vehicle accidents ${ }^{30}$ and assaults. ${ }^{31}$ The novel finding of an independent negative correlation identified between Marshall grade and PTSD invites speculation that more severe structural brain damage may exert a modest protective effect against PTSD symptoms. This is borne out in the previous literature suggesting that severe TBI may prevent the development of PTSD in some cases. For example, it has been proposed that the prolonged periods of unconsciousness may exert a protective influence. ${ }^{32}$ This may be attributable to amnesia interfering with the process by which traumatic memories are formed. While intuitively plausible, the picture is complicated by findings in patients with $\mathrm{mTBI}$, in which a longer duration of post-traumatic amnesia was found to be protective against certain PTSD symptoms in spite of the absence of overt structural brain injury. ${ }^{33}$ Some have extended this line of reasoning further to suggest that mTBI and PTSD are mutually exclusive regardless of amnesia. ${ }^{7}$

In order to reconcile the findings of these potentially conflicting studies, three main mechanisms have been proposed to link TBI with PTSD via memory systems: unimpaired traumatic memories, traumatic amnesia with spared implicit memory of trauma and islands of memory' within post-traumatic amnesia. ${ }^{34}$ The findings of this study can be recognised within this framework, as more severe brain injury findings on CT are a significant predictor of milder PTSD symptoms. In more severe TBI, structural damage (and resultant neuronal loss) may produce functional impairment of implicit memory systems. Deficits in implicit memory are not easily recognised in routine clinical assessment of post-traumatic amnesia (which essentially test declarative, but not implicit, memory). Future research into these mechanisms may benefit from avoiding a potentially arbitrary dichotomy between mild and more severe TBI. Quantifying or systematically classifying brain injury severity on a continuous basis using more sophisticated imaging may enable measurable brain injury factors to be linked to different symptoms within PTSD. A refined method based on this principle may enable information from more detailed radiological modalities (such as MRI) to predict psychiatric symptoms at a level of precision that could become clinically meaningful. In future, this may require the specific use of appropriate imaging (such as MRI) to search for relevant markers of poor long-term outcome, rather than repurposing existing scans in an opportunistic manner. In cases where a focal traumatic lesion is identified, future research may benefit from also 
exploring the effect of the anatomical location of TBI and its relationship to psychiatric symptoms. Such precision may in future enable a meaningful taxonomy of the specific psychiatric sequelae that may arise, depending on the nature of their brain injury, ${ }^{35}$ with interventions targeted accordingly.

In spite of the limitations inherent in observational study of an outpatient clinic cohort, this research illustrates that PTSD represents a common condition among people with TBI. Furthermore, routinely performed CT scans can be reviewed to identify features that relate to psychiatric morbidity in a real-world civilian population. Higher Marshall grades (eg, 5-6) are modestly associated with lower PCL-C scores. The presence of a relationship between more severe brain injury and milder PTSD symptoms represents a novel finding, given that depression and PCSs have been controlled for in this design. The implications of this extend from the theoretical to the practical-inviting further exploration using more sophisticated imaging, as well as pointing toward pragmatic approaches to screen those patients with TBI at the highest risk of PTSD.

\section{Author affiliations}

${ }^{1}$ Department of Psychiatry, School of Psychology and College of Medical and Dental Sciences, University of Birmingham, Birmingham, UK

${ }^{2}$ National Institute for Health Research, Surgical Reconstruction and Microbiology

Research Centre, Queen Elizabeth Hospital, Birmingham, UK

${ }^{3}$ Department of Radiology, Queen Elizabeth Hospital, Birmingham, UK

${ }^{4}$ Institute of Inflammation and Aging, University of Birmingham, Birmingham, UK

Contributors KLQ was involved in study design, reviewing collected data, performing the analyses and drafting the manuscript. RU was involved in study design, developing analytic strategy and also provided extensive commentary on the manuscript. ET was involved in data collection, assisted with study design and provided extensive commentary on the manuscript. DJD, VS and AB were involved in the development of the database on which the study is based and drafting, editing and approving the manuscript.

Funding The authors have not declared a specific grant for this research from any funding agency in the public, commercial or not-for-profit sectors.

Competing interests None declared.

Patient consent for publication Not required.

Ethics approval Approval was granted under NHS Research Ethics (HRA 17/ LO/0153).

Provenance and peer review Not commissioned; externally peer reviewed.

Data sharing statement Data underlying results can be applied for via the corresponding author after deidentification. Full access to the patient database will not be available.

Open access This is an open access article distributed in accordance with the Creative Commons Attribution Non Commercial (CC BY-NC 4.0) license, which permits others to distribute, remix, adapt, build upon this work non-commercially, and license their derivative works on different terms, provided the original work is properly cited, appropriate credit is given, any changes made indicated, and the use is non-commercial. See: http://creativecommons.org/licenses/by-nc/4.0/.

\section{REFERENCES}

1. Kessler RC, Sonnega A, Bromet E, et al. Posttraumatic stress disorder in the national comorbidity survey. Arch Gen Psychiatry 1995;52:1048-60.

2. Tarrier N, Gregg L. Suicide risk in civilian PTSD patients. Soc Psychiatry Psychiatr Epidemiol 2004;39:655-61.
3. Hidalgo RB, Davidson JR. Posttraumatic stress disorder: epidemiology and health-related considerations. J Clin Psychiatry 2000;61 Suppl 7:5-13.

4. American Psychiatric Association. Diagnostic and Statistical Manual of Mental Disorders (5th edition) (DSM-5): APA, 2013.

5. World Health Organization. The ICD-10 classification of mental and behavioural disorders: clinical descriptions and diagnostic guidelines. Geneva 1992.

6. Mayou R, Bryant B, Duthie R. Psychiatric consequences of road traffic accidents. BMJ 1993;307:647-51.

7. Sbordone RJ, Liter JC. Mild traumatic brain injury does not produce post-traumatic stress disorder. Brain Inj 1995;9:405-12.

8. Stein MB, McAllister TW. Exploring the convergence of posttraumatic stress disorder and mild traumatic brain injury. Am J Psychiatry 2009;166:768-76.

9. Bryant RA, Marosszeky JE, Crooks J, et al. Posttraumatic stress disorder after severe traumatic brain injury. Am J Psychiatry 2000;157:629-31.

10. Bryant RA, Harvey AG. Postconcussive symptoms and posttraumatic stress disorder after mild traumatic brain injury. J Nerv Ment Dis 1999;187:302-5.

11. Levin HS, Wilde E, Troyanskaya M, et al. Diffusion tensor imaging of mild to moderate blast-related traumatic brain injury and its sequelae. J Neurotrauma 2010;27:683-94.

12. Bigler ED, Maxwell WL. Neuropathology of mild traumatic brain injury: relationship to neuroimaging findings. Brain Imaging Behav 2012;6:108-36.

13. Lew HL, Vanderploeg RD, Moore DF, et al. Overlap of mild TBI and mental health conditions in returning OIF/OEF service members and veterans. J Rehabil Res Dev 2008;45:11.

14. Schneiderman Al, Braver ER, Kang HK. Understanding sequelae of injury mechanisms and mild traumatic brain injury incurred during the conflicts in Iraq and Afghanistan: persistent postconcussive symptoms and posttraumatic stress disorder. Am J Epidemiol 2008;167:1446-52.

15. Langlois JA, Rutland-Brown W, Wald MM. The epidemiology and impact of traumatic brain injury: a brief overview. J Head Trauma Rehabil 2006;21:375-8.

16. Carlson KF, Kehle SM, Meis LA, et al. Prevalence, assessment, and treatment of mild traumatic brain injury and posttraumatic stress disorder: a systematic review of the evidence. J Head Trauma Rehabil 2011;26:103-15

17. Marshall LF, Marshall SB, Klauber MR, et al. A new classification of head injury based on computerized tomography. J Neurosurg 1991;75:S14-S20.

18. von Steinbuechel N, Petersen C, Bullinger M. QOLIBRI Group. Assessment of health-related quality of life in persons after traumatic brain injury--development of the Qolibri, a specific measure. Acta Neurochir Supp/ 2005;93:43.

19. King NS, Crawford S, Wenden FJ, et al. The rivermead post concussion symptoms questionnaire: A measure of symptoms commonly experienced after head injury and its reliability. J Neurol 1995;242:587-92.

20. Kroenke K, Spitzer RL. The PHQ-9: A new depression diagnostic and severity measure. Psychiatr Ann 2002;32:509-15.

21. Wilkins KC, Lang AJ, Norman SB. Synthesis of the psychometric properties of the PTSD checklist (PCL) military, civilian, and specific versions. Depress Anxiety 2011;28:596-606.

22. Weathers F, Litz B, Herman D, et al. The PTSD Checklist: Reliability, validity, \& diagnositic utility. Annual Meeting of the Internation Society of Traumatic Stress Studies. San Antonio, Texas, 1993.

23. Hoge CW, McGurk D, Thomas JL, et al. Mild traumatic brain injury in U.S. Soldiers returning from Iraq. N Engl J Med 2008;358:453-63.

24. Terhakopian A, Sinaii N, Engel CC, et al. Estimating population prevalence of posttraumatic stress disorder: An example using the PTSD checklist. J Trauma Stress 2008;21:290-300.

25. Udekwu P, Kromhout-Schiro S, Vaslef S, et al. Glasgow Coma Scale score, mortality, and functional outcome in head-injured patients. $J$ Trauma 2004;56:1084-9.

26. Cifu DX, Keyser-Marcus L, Lopez E, et al. Acute predictors of successful return to work 1 year after traumatic brain injury: A multicenter analysis. Arch Phys Med Rehabil 1997;78:125-31.

27. Keane TM, Wolfe J. Comorbidity in post-traumatic stress disorder an analysis of community and clinical studies1. J Appl Soc Psychol 1990;20:1776-88.

28. Ruggiero KJ, Del Ben K, Scotti JR, et al. Psychometric properties of the PTSD Checklist-Civilian Version. J Trauma Stress 2003;16:495-502.

29. Qureshi Al, Malik AA, Adil MM, et al. Hematoma enlargement among patients with traumatic brain injury: Analysis of a prospective multicenter clinical trial. J Vasc Interv Neurol 2015;8:42. 
30. Holeva V, Tarrier N, Wells A. Prevalence and predictors of acute stress disorder and PTSD following road traffic accidents: Thought control strategies and social support. Behav Ther 2001;32:65-83.

31. Kilpatrick DG, Ruggiero KJ, Acierno R, et al. Violence and risk of PTSD, major depression, substance abuse/dependence, and comorbidity: Results from the National Survey of Adolescents. $J$ Consult Clin Psychol 2003;71:692-700.

32. Glaesser J, Neuner F, Lütgehetmann R, et al. Posttraumatic stress disorder in patients with traumatic brain injury. BMC Psychiatry 2004:4:1.
33. Bryant RA, Creamer M, O'Donnell M, et al. Post-traumatic amnesia and the nature of post-traumatic stress disorder after mild traumatic brain injury. J Int Neuropsychol Soc 2009;15:862-7.

34. King NS. Post-traumatic stress disorder and head injury as a dual diagnosis: "islands" of memory as a mechanism. Journal of Neurology, Neurosurgery \& Psychiatry 1997;62:82-4.

35. Finset A, Andersson S. Coping strategies in patients with acquired brain injury: relationships between coping, apathy, depression and lesion location. Brain Inj 2000;14:887-905. 